\title{
Abogacía para el control del tabaco en México: retos y recomendaciones
}

Jesús Felipe González-Roldán, MD, MSP, M en Admón Púb.(')

E 1 tabaco es un producto originario del continente americano, que en el siglo XVI fue exportado a Europa, desde donde su uso se extendió al resto del mundo. En México existen evidencias literarias que documentan su utilización de manera importante por parte de los pueblos prehispánicos. Desde la Revolución industrial, su uso como cigarro, puro, pipa o en otras formas se ha generalizado en todos los países, a pesar de que desde hace varias décadas se sabe que es una de las principales causas prevenibles de enfermedad y muerte y de que conocemos que la nicotina es una sustancia que produce adicción.

En nuestro país es cada vez más común la elaboración y consumo de distintas gamas de puros con la hoja de tabaco, que ha cobrado auge en los últimos cinco años por cuestiones de estatus y moda. Sin embargo, la principal forma de consumo del tabaco sigue siendo a través del cigarro labrado, cuya industria y mercado están bajo el dominio de dos compañías tabacaleras trasnacionales: Philip Morris México (PMM) y British American Tobacco (BAT).

Desde octubre de 2007, se discutieron en México iniciativas legislativas que tienden a regular distintos aspectos para el control del tabaco. Por un lado, en el ámbito federal, se aprobó en las cámaras de Diputados y de Senadores la Ley General para el Control del Tabaco -aún pendiente de ser emitida por el Poder Ejecutivo-, la cual establece un marco normativo general para distintos aspectos que tienen que ver con la producción, co- mercialización, distribución, venta y consumo de tabaco. Por otra parte, el 26 de febrero de 2008 fue aprobada por la Asamblea Legislativa del Distrito Federal, la Ley de Protección a la Salud de los No Fumadores, la cual establece espacios 100\% libres de humo en toda oficina pública o privada cerrada, en todo el transporte público y en establecimientos mercantiles como restaurantes, bares, discotecas, billares y boliches.

Habría que señalar que los poderes Ejecutivo y Legislativo, tanto del ámbito federal como en algunos locales, fundamentalmente en el Distrito Federal, han tenido que manifestar sus posiciones respecto de la propuesta de Ley Federal para el Control del Tabaco y de las modificaciones que se plantearon a la Ley de Protección a la Salud de los No Fumadores, así como a la Ley para el Funcionamiento de Establecimientos Mercantiles, ambas de aplicación en la capital del país. El tema se plantea, analiza y discute en oficinas, clubes, restaurantes, bares, y hogares de nuestro país. Este hecho es por sí mismo relevante, pues ha permitido ventilar de manera abierta temas de interés público.

El debate sobre los principios éticos y de prevención de enfermedades que se encuentran implícitos en el establecimiento de todos los espacios públicos como 100\% libres de humo de tabaco no es algo nuevo. En los países y ciudades que han emprendido medidas similares la discusión se ha visto alimentada al mismo tiempo por el legítimo derecho de los fumadores a manifestar su posición al respecto $\mathrm{y}$, esto en mayor medida, por

(I) Presidente de la Red México sin Tabaco. Secretario y Socio Fundador de ACTA AC, México. 
las estrategias empleadas por una industria tabacalera poderosa en influencia política y económica.

Hace seis años, Stanton Glantz, responsable de que la industria tabacalera (IT) tuviera que hacer de conocimiento público sus documentos internos, y J. Barnoya explicaron de manera clara, al analizar dichos documentos, las diversas estrategias empleadas por la IT para detener y contrarrestar el establecimiento de ambientes libres de humo en Latinoamérica. ${ }^{1}$ En su análisis encontraron evidencia sobre las siguientes acciones:

a) Cabildeo con legisladores y funcionarios para bloquear, detener o modificar iniciativas.

b) Confrontar a fumadores contra no fumadores.

c) Utilización de cámaras, asociaciones, agrupaciones y/o sindicatos afines o relacionados comercialmente con la IT.

d) Reclutamiento, entrenamiento y manejo mediático de consultores y líderes de opinión.

e) Captar o promover el apoyo por parte de líderes de opinión e "intelectuales".

f) Conformación de grupos de fumadores.

g) Desviar la atención del daño producido por el humo de tabaco hacia otros contaminantes.

h) Empleo de argumentación del respeto a las libertades humanas.

i) Promover recursos jurídicos.

j) No exponerse de manera pública y abierta.

Sin lugar a dudas, todas estas acciones se han utilizado en mayor o menor grado en los recientes procesos que se han vivido en México.

\section{El tabaquismo en México}

El tabaquismo se ha convertido en la principal causa prevenible de enfermedad y muerte en el mundo. En México, ocasiona más de 60000 muertes anuales y estimaciones de la Secretaría de Salud señalan un costo cercano a los 30 mil millones de pesos anuales para el Sistema Nacional de Salud, como consecuencia de la atención de padecimientos ligados a este hábito.

Las cifras de la Encuesta Nacional de Adicciones (ENA) de 2002 señalan una prevalencia de $26.4 \%$ en la población urbana que cuenta entre 12 y 65 años de edad, donde $39 \%$ de los hombres y $16 \%$ de las mujeres fuman. Sin embargo, en la reciente Encuesta Mundial de Tabaquismo y Juventud, capítulo mexicano, realizada en escuelas secundarias de diversas ciudades del país, se presentan ya datos donde la relación entre hombres y mujeres que fuman es igual.
Las tendencias epidemiológicas en México son las siguientes:

a) Estabilidad en la prevalencia de consumo. Durante los últimos 20 años se ha visto que el número de personas fumadoras, si bien se ha incrementado en número, se ha mantenido estable en cuanto a la relación poblacional, lo cual significa que, en asentamientos urbanos, 1 de cada 4 personas mayor de 12 años fuma.

b) Inicio en el consumo a más temprana edad. La edad de inicio ha disminuido, ubicándose actualmente entre los 12 y 13 años de edad, por lo que cada vez los adolescentes tienen mayor propensión a consumir otro tipo de drogas (lícitas e ilícitas), ya que el tabaco ha demostrado ser la droga de inicio para el consumo de otras, como el alcohol, la marihuana o la cocaína.

c) Incremento de consumo en las mujeres. En los últimos 20 años se ha observado de manera significativa que el incremento mayor en el consumo de tabaco se ha dado entre las mujeres, y la relación entre hombre/mujer fumadora se ha reducido de 10/1 en $1998^{2}$ a 2/1 en 2002, y en estudiantes de secundaria es de $1 / 1 .^{3}$

d) Mayor consumo entre adolescentes. De igual forma, si bien las encuestas nacionales señalan que 1 de cada 10 adolescentes fuma cotidianamente, las últimas encuestas realizadas entre estudiantes de secundaria señalan un consumo mayor de casi $20 \%$ de adolescentes fumadores. ${ }^{4}$

\section{El Convenio Marco para el Control del Tabaco ${ }^{5}$}

La Organización Mundial de la Salud (OMS) desarrolló el primer convenio internacional de salud pública orientado a que los países adoptaran un compromiso global y establecieran medidas mínimas para el control del tabaquismo en el Mundo, para de esta forma enfrentar de manera conjunta la pandemia que el tabaco está ocasionando.

México fue el primer país de Latinoamérica en firmarlo (decimoséptimo a nivel mundial) el 12 de agosto de 2003, siendo ratificado por el Senado de la República el 14 de abril de 2004 y publicado en el Diario Oficial de la Federación el 12 de mayo de 2004, con lo que entró en vigor al día siguiente de su publicación. ${ }^{6}$ De manera desafortunada, México suscribió el 18 de junio de 2004 un acuerdo con la IT que impidió, de manera significativa, cualquier avance regulatorio y de control al tabaco. 
Actualmente, bajo el Convenio Marco para el Control del Tabaco existen medidas mínimas que México debe impulsar, tales como:

a) Propiciar espacios $100 \%$ libres de humo.

b) Instrumentar una política fiscal que incremente de manera significativa el precio de los cigarros $y$, con ello, dificulte la adquisición de éstos.

c) Prohibición completa de la publicidad, promoción y patrocinio.

d) Establecer medidas para el empaquetado y etiquetado de los productos.

e) Atender el comercio ilícito.

f) Desarrollar y fomentar campañas educativas.

\section{La IT en México}

Es necesario conocer y entender el comportamiento de la IT para poder impulsar de manera adecuada políticas públicas que conlleven la protección de la salud de la población por encima de cualquier interés económico o político.

El consumo de cigarros en México es anualmente de 48905 millones, lo que nos ubica en el décimoquinto lugar mundial, con un mercado nacional aproximado de 30000 millones de pesos (2006), de los cuales las compañías tabacaleras destinan 4000 millones de pesos en gastos de promoción, publicidad, patrocinios y relaciones gubernamentales.

Si bien hace 10 años la distribución del mercado era casi homogénea, actualmente Philip Morris MéxicoCigatam controla $63 \%$ del mercado, mientras que British American Tobacco $36 \%$ y el restante $1 \%$ es de otras compañías con poca presencia en México (figura 1).

Estas dos empresas constituyen el denominado Consejo Nacional de la Industria Tabacalera (Conainta), el cual es utilizado fundamentalmente para acciones de abogacía y cabildeo ante el Poder Ejecutivo, sobre todo en su ámbito hacendario, agrícola, económico y de regulación sanitaria, y ante los poderes legislativos, tanto federal como locales. Es a través de este Consejo que la industria busca mantener su status quo, al tratar de detener o bloquear iniciativas que ésta considera potencialmente perjudiciales.

\section{Philip Morris México-Cigatam}

La principal empresa en ventas de nuestro país tiene una manera peculiar de operar en México, a través de dos empresas perfectamente articuladas y relacionadas entre sí. Por una parte está Cigatam, como empresa encargada del proceso de fabricación, y por la otra Phillip Morris México (PMM), como empresa destinada a la comercia-

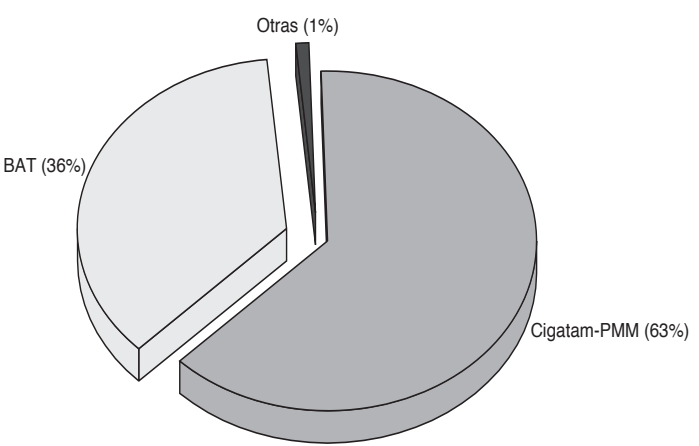

Fuente: Reporte Anual de Grupo Carso al 3I de diciembre de 2006 y datos de BAT

Figura I. Industria tabacalera. Participación de merCADO NACIONAL (2006)

lización y manejo mercadológico de sus marcas. Como propietario fundamental de ellas, aparece Grupo Carso, uno de los consorcios empresariales más importantes en el ámbito mundial, con sede en México.

Grupo Carso es propietario de $50.01 \%$ de Cigatam y de $49.99 \%$ de PMM (recientemente anunciaron la venta de $30 \%$ de sus acciones de PMM). En el reporte anual 2006, que Grupo Carso presenta ante la Bolsa Mexicana de Valores, se señala que Cigatam tuvo ventas por 16 859 millones de pesos, con una utilidad bruta de 1170 millones de pesos. ${ }^{7}$

Debido a su influencia económica y política en nuestro país, dicha empresa es la que realiza de manera preponderante las acciones de cabildeo en nombre de la IT.

Así, en el reciente debate legislativo relativo a la aprobación de la Ley General para el Control del Tabaco, manifestaron mediante una comunicación privada dirigida a las comisiones de Salud de las cámaras de Senadores y de Diputados, su beneplácito por que el modelo a seguir respecto de los espacios libres de humo de tabaco es el que España instrumentó. Coincidentemente, éste fue el modelo propuesto públicamente por todas aquellas personas fumadoras que dieron su opinión ante los diversos foros y medios de comunicación.

\section{British American Tobacco}

Tiene su sede empresarial en la ciudad de Monterrey, Nuevo León, y cuenta con presencia en México a partir de que, en 1997, adquirió lo que en ese entonces era Cigarrera La Moderna. 
Poseía, hasta 2006, sólo 36\% del mercado de cigarros en México. Su participación de mercado ha venido disminuyendo de manera paulatina, debido sobre todo a la pulverización que ha realizado la empresa desde el punto de vista de mercadeo y de negocios de sus marcas nacionales que tenían mayor participación en el mercado mexicano.

Actualmente cuentan, según datos de la empresa, con poco más de 1850 empleados y ofrecen 15 marcas en México, cada una bajo diversas presentaciones, a través de sus más de 180000 puntos de venta en la República mexicana. Su producción anual es de más de 33 mil millones de cigarros, de los cuales solamente 18 mil millones son para venta en el mercado nacional y el resto están destinados principalmente hacia el mercado canadiense.

Lo anterior es relevante en virtud de que en la argumentación respecto del establecimiento de pictogramas en las cajetillas y paquetes de cigarros, la IT ha señalado que hay dificultad tecnológica para su instrumentación; sin embargo las cajetillas que dicha empresa elabora en México para su venta en Canadá contemplan dichos pictogramas.

Por otra parte, la empresa es responsable desde hace por lo menos 10 años de impulsar el programa "Convivencia en Armonía", que busca establecer lugares para fumadores en sitios públicos cerrados de restaurantes, centros de trabajo y universidades. En el sitio de internet que la compañía dedica a este tema se encuentran aseveraciones que podrían sustentar políticas públicas para mantener espacios públicos cerrados $100 \%$ libres del humo de tabaco:

- "Los lugares llenos de humo representan un motivo de gran desaliento, tanto para los clientes de un establecimiento, como para el personal que labora en el mismo".

- "La mayoría de las personas consideran que los centros de consumo, tales como restaurantes, cafeterías, bares, casinos, clubes nocturnos y privados deberían de esforzarse más por ofrecer y garantizar un aire más limpio".

- "Por lo general, en los lugares donde se venden alimentos y bebidas, los clientes desean una mejor ventilación y áreas de no fumar".

- "El personal a menudo simpatiza con las prohibiciones a fumar y prefiere, por mucho, trabajar en ambientes donde sus prendas y cabello no huelan a humo de cigarro".

Una preocupación importante de esta empresa, relacionada con la caída en ventas que ha padecido, ha sido la prohibición completa de la publicidad, promoción y patrocinio, por lo que emprendió en su momento una estrategia de cabildeo para posicionar sus argumentos al respecto entre legisladores federales del estado de Nuevo León.

\section{De las instituciones a la sociedad civil organizada}

Hoy la sociedad mexicana se encuentra inmersa en un debate de salud pública que nos concierne a todos y permite que diversos sectores se pronuncien de manera cotidiana sobre cómo disminuir la afectación y el daño que el tabaco ocasiona. Por otra parte en los últimos años, a pesar de que se contaba con evidencia científica, los avances en materia de políticas públicas se habían dado de manera paulatina.

Si bien el seguimiento de la epidemia del tabaquismo en México está perfectamente documentado y se cuenta con diversos estudios epidemiológicos que permiten identificar el comportamiento del tabaquismo, la posibilidad de instaurar políticas eficaces de control había sido limitada y desarticulada. De igual manera, se han realizado estudios en cuanto al cumplimiento de la ley donde, por ejemplo, Kuri y colaboradores ${ }^{8}$ han demostrado que la venta de tabaco a menores de edad y la venta de cigarros por unidad es una constante presente en nuestro entorno, a pesar de que existen las prohibiciones respectivas. Sin embargo, esta evidencia no ha bastado para que el área responsable de hacer cumplir la ley en este aspecto actúe.

El tema del control del tabaco en México ha estado presente en el ámbito del Poder Ejecutivo desde hace por lo menos 20 años, como lo prueba el Comité Interinstitucional para el Combate del Tabaquismo, que agrupa a representantes de diversas instituciones, sobre todo de salud, que tienen interés o responsabilidad en cuanto al combate al tabaquismo. La finalidad primordial del Comité ha sido la de organizar las actividades alrededor del Día Mundial sin Fumar, bajo la coordinación del Instituto Nacional de Enfermedades Respiratorias (INER) y el Consejo Nacional contra las Adicciones (Conadic).

Sin embargo, para fines de 1997, el entonces Secretario de Salud encomendó a un selecto grupo de actores sociales la realización de actividades de abogacía en el control del tabaco, iniciativa de la cual surgió el Grupo Catalizador para el Combate del Tabaquismo, que contaba con la participación de diversos miembros del Comité Interinstitucional y se encargó de impulsar políticas públicas para la prevención y control del tabaquismo en el país. Dicho grupo sesionaba de manera semanal y se caracterizó fundamentalmente por la interdisciplinariedad y la pluralidad de sus integrantes, quienes al ver que con el cambio de administración quedaba disuelto, decidieron constituirse en sociedad civil. Así, el 28 de 
abril de 2003 se creó la Alianza Contra el Tabaco (ACTA $\mathrm{AC}$ ), cuya filosofía y principios se basan en impulsar el establecimiento de políticas públicas que controlen la oferta y la demanda del tabaco en México.

ACTA pretende informar, orientar, concienciar y capacitar a la población en general, al personal de salud, a funcionarios y legisladores respecto de la importancia que reviste para nuestro país lograr ambientes 100\% libres de humo de tabaco e instrumentar políticas sanitarias que coadyuven a abatir de manera importante las repercusiones negativas que para la salud y la economía representa el tabaquismo.

\section{El Reporte de la Sociedad Civil}

Desde mayo de 2006, ACTA, en coordinación con la Fundación Interamericana del Corazón (FIC) y con el apoyo de la Asociación Canadiense de Salud Pública (CPHA), ha desarrollado de manera anual un Reporte de la Sociedad Civil sobre los avances que nuestro país registra en el cumplimiento del CMCT que signó ante la OMS y que fue ratificado por el Senado de la República el 14 de abril de 2004.

Este Reporte constituye una valiosa herramienta para que comunicadores, decisores, legisladores, investigadores y población interesada en el tema conozcan los avances normativos y operativos que han sucedido en nuestro país.

Esta organización debe considerarse clave en el actual proceso de elaboración de leyes que vive en nuestro país, y su autonomía le ha permitido denunciar casos como el convenio de la Secretaría de Salud con la industria tabacalera en 2004, que impidió el avance regulatorio en materia de control del tabaco.

Asimismo, además de participar en 2005 en el Primer Foro sobre el Tabaquismo, organizado de manera conjunta con la Comisión de Salud de la Cámara de Diputados, en mayo de 2006 y en mayo de 2007 se presentaron, de manera respectiva, el Primer y Segundo Reporte de la Sociedad Civil sobre la instrumentación del $\mathrm{CMCT}$ en México. Estos reportes se presentan ante los medios de comunicación y tienen por objeto difundir los avances, rezagos e interferencias que han existido en nuestro país para el cumplimiento de lo plasmado en el CMCT.

Con este instrumento, además de difundir los aspectos relevantes del CMCT, se ha podido informar y orientar a quienes toman las decisiones, en los ámbitos Ejecutivo y Legislativo, y a comunicadores e investigadores sobre las tareas pendientes en el control del tabaco.

Cabe señalar que, si bien ONG de Canadá y Francia han elaborado reportes similares, el presentado en México, es el único reporte mundial elaborado anualmente sobre el tema, por lo que ha sido reconocido por la Framework Convention Alliance (FCA), como un buen ejemplo para otros países.

La divulgación que sobre este tema han hecho los medios de comunicación, tanto televisión como radio pero, sobre todo, prensa escrita, ha sido fundamental, tanto para orientar y sensibilizar a la sociedad, como para, en su momento, denunciar incongruencias o hechos poco claros respecto de la relación de la IT con autoridades como la Comisión Nacional para la Protección contra Riesgos Sanitarios (COFEPRIS).

\section{El reciente impulso legislativo}

Cabe señalar que la firma del convenio que la Secretaría de Salud realizó en 2004 con la IT significó un parteaguas e intensificó las acciones de abogacía y cabildeo que la sociedad civil mexicana ha emprendido en este campo, dado que se hicieron visibles los intereses de la industria y manifiesta su intención de comprar voluntades de los funcionarios públicos, como fue el caso de la denuncia que realizó un diputado en 2005, sobre la forma en que la IT ofreció viajes a diversos diputados para buscar apoyo e inhibir iniciativas legislativas que pudieran afectarles.

Ahora bien, con el cambio de gobierno hubo también un impulso de las políticas públicas en materia de tabaco desde el sector salud. En mayo de 2007 se interrumpió el convenio que la Secretaría de Salud sostenía con la IT, con lo que tácitamente se aceptaba que dicho acuerdo nunca debió existir. Durante la Segunda Conferencia de los Países Signatarios del Convenio Marco, celebrada del 30 de junio al 6 de julio de 2007 en Bangkok, Tailandia, México informó que había dado por concluido dicho convenio y que recomendaba que los demás países no realizaran en lo futuro este tipo de acuerdos. $^{9}$

En octubre del mismo 2007, se presentaba de manera conjunta por los presidentes de las comisiones de Salud de las cámaras de Diputados y de Senadores, la iniciativa para impulsar una Ley General para el Control del Tabaco, buscando cumplir con los acuerdos señalados por parte del CMCT.

Por otra parte, en la Asamblea Legislativa del Distrito Federal se presentaba una propuesta de modificación a la Ley de Protección a los No fumadores, que aprobada de manera mayoritaria establecía de manera inicial el que restaurantes, bares, discos, cantinas y billares deberían dedicar $70 \%$ de su espacio a no fumadores y que el 30\% restante se podría destinar, con medidas de 
aislamiento, como espacio para fumadores.

El surgimiento de un amparo contra dicha modificación por parte de un importante grupo restaurantero y las manifestaciones hechas por parte de la Cámara Nacional de la Industria Restaurantera (Canirac) dieron origen a un debate profundo en medios de comunicación respecto de cómo llevar a cabo dichas separaciones.

Recientemente, en México se aprobaron de manera histórica y en la misma fecha dos instrumentos jurídicos que buscan retomar el liderazgo que se dejó de ejercer en la materia. El pasado 26 de febrero de 2008, la Cámara de Senadores aprobó la Ley General para el Control del Tabaco y la Asamblea Legislativa del Distrito Federal aceptó las modificaciones planteadas a la Ley de Protección a los No Fumadores, para establecer que todo espacio cerrado público sea considerado como área $100 \%$ libre de humo de tabaco.

En ambos procesos fue fundamental considerar los siguientes elementos como aspectos relevantes para impulsar la aprobación de dichas leyes:

\section{a) Información estadística del tema}

Contar con un seguimiento puntual del comportamiento epidemiológico del tabaquismo en México fue sin duda alguna la herramienta fundamental para presentar el tema de manera sólida ante los legisladores, tanto federales como asambleístas del DF. Fue importante saber los datos duros del costo sanitario y social de este padecimiento y cuáles debían ser las principales políticas públicas a impulsar.

También fueron fundamentales los datos de una encuesta de opinión, realizada por el Consejo Mexicano Contra el Tabaquismo (CMCT) a través de la empresa Parametría, para señalar ante funcionarios y legisladores que 7 de cada 10 personas fumadoras estaban de acuerdo con la ley y que en términos generales 8 de cada 10 personas apoyaban la regulación de espacios 100\% libres de humo.

\section{b) Conocimiento del comportamiento de la IT en México y otras partes del mundo}

Contar con el antecedente de la intervención por parte de la IT en México de manera muy abierta, con el convenio suscrito con la Secretaría de Salud federal en 2004, permitió contar con el apoyo de diversas instancias internacionales, que forman parte de la FCA, para conocer las experiencias de intervención que han seguido en lugares como Argentina y Panamá. Por ejemplo, para conocer de manera puntual el modo en que la IT mantiene negociaciones en el ámbito privado a través de sus áreas de relaciones gubernamentales y agencias de cabildeo, y utiliza a otros actores como cámaras restauranteras, asociaciones de fumadores y/o líderes de opinión para argumentar en lo público la defensa de sus intereses.

En los recientes procesos legislativos, federal y local en el DF, ha sido evidente cómo a pesar de ser el principal actor involucrado en el proceso de reformas, la IT estuvo ausente de manera directa del debate público y utilizó a otros actores para que salieran a defender sus posiciones.

\section{c) Introducción del tema en la agenda pública} del Ejecutivo y el Legislativo

Las acciones de cabildeo con el Ejecutivo y Legislativo han sido fundamentales para impulsar modificaciones que realmente conlleven a promover un efectivo control del tabaco en México.

Es de reconocer que tanto para el Secretario de Salud federal, como para el Secretario de Salud del Distrito Federal, el tema fue puesto como una prioridad en su agenda pública y por ende el apoyo que ellos y demás funcionarios de sus áreas brindaron fue decisivo para impulsar en el ámbito legislativo la promulgación de estas leyes.

El papel del Ejecutivo (Secretaría de Salud), requirió en primera instancia de poner en sintonía a los principales actores al interior. Fue relevante el rol que asumieron la Secretaría de Salud y el Conadic, quienes de manera pública y en diversos foros convocaron a apoyar este proceso legislativo y cuyos funcionarios de manera personal se reunieron y proporcionaron información a actores relevantes, sobre todo legisladores, independientemente del partido político o ideología que éstos tuvieran.

En cuanto a la propuesta legislativa presentada por el Partido Nueva Alianza en el Distrito Federal, ésta fue apoyada de manera unánime por los tres principales partidos políticos: PAN, PRD y PRI. El apoyo del secretario de salud del DF fue también definitivo para contrarrestar la argumentación y oposición presentados por una minoría de diputados del PSD y del PRD, quienes utilizaban la misma argumentación que la IT había hecho llegar desde octubre de 2007 a las comisiones de Salud de ambas cámaras federales y a la propia Asamblea Legislativa. Cabe señalar que de manera inicial ellos habían votado a favor de establecer espacios libres de humo.

Por lo que respecta al papel de la sociedad civil, es de resaltar que diversas asociaciones, como ACTA, FIC México, Consejo Mexicano Contra el Tabaco, Asociación Mexicana de Lucha Contra el Cáncer y la Red México sin Tabaco, establecieron durante todo 2007 y el presente 
año reuniones constantes tanto con funcionarios de la Secretaría de Salud, como con legisladores, para impulsar que México cumpla con los compromisos contraídos a través del CMCT en la lucha contra el tabaquismo.

d) Seguimiento de las discusiones e identificación de actores principales en la toma de decisiones

Un elemento clave para la consecución de la aprobación de las leyes fue conocer de manera puntual las principales líneas discursivas en las cuales se centró el debate y quiénes eran los principales actores, tanto por su posición a favor como en contra de las iniciativas.

De este modo se visualizó que en ambas leyes, federal y local, el debate se circunscribió de manera fundamental al establecimiento de espacios 100\% libres de humo y, adicionalmente, en el caso de la Ley General para el Control del Tabaco, en cuanto a las restricciones en el empaquetado, etiquetado y aspectos publicitarios del producto.

\section{e) Estrategia comunicacional}

Una parte importante fue provocar y obtener el apoyo de la sociedad para las leyes en cuestión, por lo que se requirieron diversas acciones comunicacionales tendientes a informar, orientar y en su caso movilizar a la sociedad en apoyo, sobre todo, al establecimiento de espacios cerrados $100 \%$ libres de humo.

Se planteó la necesidad de realizar e instrumentar lo siguiente:

1. Monitoreo y análisis de notas informativas: es necesario conocer el sentir de los medios y en su caso dar respuesta oportuna a notas que se considere que lo ameritan, por lo que se realizó un monitoreo diario y se analizaron las diversas notas y editoriales que se han venido manejando respecto a estas leyes en:

- Televisión

- Radio

- Revistas y periódicos

2. Elaboración y difusión de mensajes:

- Para apoyar la aprobación de la Ley de Protección a los No Fumadores en el DF se realizó y distribuyó previo a la sesión de discusión en la Asamblea Legislativa diverso material gráfico (folletos, postres, pendones, tarjetas postales) de la campaña "Se respira respeto" (figura 2).

- De igual forma se logró, con apoyo de la World Lung Foundation, obtener fondos para la elaboración de tres promocionales de radio, los cuales se transmitieron durante el mes de abril,

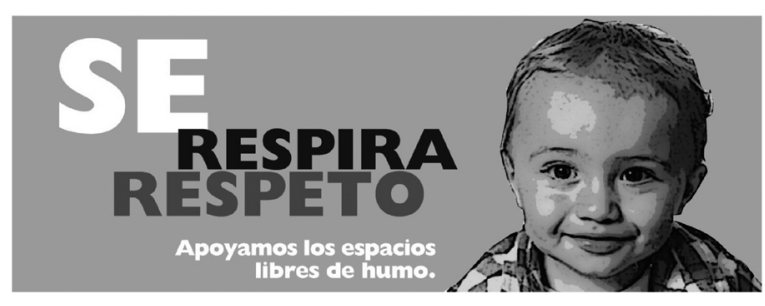

Figura 2. Campaña publicitaria para el apoyo ciudadano a la MOdificación de La Ley de Protección de los No fumadores EN EL DF EN EL 2008

una vez que entró en vigor la ley en el DF, para promover el cumplimiento de la normativa en los espacios públicos cerrados $100 \%$ libres de humo de tabaco. Se transmitieron a través de tres grupos radiodifusores de alta audiencia: Grupo Radio Mil, Televisa Radio y Grupo Radio Fórmula.

3. Evaluación de contenidos:

- Se realizaron y analizaron diversas encuestas de opinión y estudios con grupos focales para definir los aspectos fundamentales donde el debate estaba concentrado y poder elaborar los argumentos de defensa que pudieran rebatir lo que la IT a través de editorialistas, líderes de opinión y/o cámaras y asociaciones argumentan en contra de estas leyes.

- Derivado de esta evaluación de contenidos se realizaron mensajes para por lo menos los siguientes grupos (hombres y mujeres):

- Trabajadores de restaurantes, bares y discotecas

- Fumadores

- No fumadores

- Propietarios de establecimientos

IV. Participación en entrevistas, programas de debates y elaboración de artículos:

- Se estableció como parte importante de la estrategia comunicacional que cualquier integrante de la coalición de la sociedad civil estuviera preparado para participar en medios y de igual forma se priorizó fomentar la elaboración de artículos de opinión para contrarrestar y centrar el debate, priorizando los aspectos relativos a la salud pública por encima de los intereses políticos y económicos.

V. Conferencias de prensa: 
- Una parte clave para lograr la sinergia y tener a los periodistas constantemente informados fue realizar reuniones con medios para presentar los diversos aspectos que el control del tabaco requiere y por qué son éstos necesarios de impulsar, dando a conocer los datos epidemiológicos y los costos sociales y económicos que el tabaquismo trae a la sociedad.

\section{f) Acción y reacción inmediata}

Dar seguimiento al modo en que se iban dando y discutiendo ambas leyes propició que existiera una reacción inmediata ante cualquier intento que buscara inhibir o congelar alguna normativa.

Dado que durante enero y febrero de 2008 el debate en los medios de difusión se centró en la Ley de Protección a los No Fumadores en el DF, el trabajo fundamental se encaminó a defender el establecimiento de espacios 100\% libres de humo.

Llamó poderosamente la atención la argumentación que se escuchó en voz de varias personalidades de nuestra sociedad, en defensa de su derecho a fumar, arguyendo que se les estaba prohibiendo hacerlo.

De de manera pública conminaron a que no dejáramos que se aplicaran políticas propias de países del primer mundo, sino que se debiera aplicar el modelo español, que permite que existan restaurantes y bares exclusivos para fumadores. De manera privada, esta misma argumentación era sostenida por un alto ejecutivo de Philip Morris México, en su escrito de octubre de 2007, enviado a la Comisión de Salud de la Cámara de Diputados.

Existían otros modelos, como el italiano, el francés, el neoyorkino y el californiano, o bien los de países latinoamericanos como Panamá, Uruguay o Argentina, pero tenían el defecto de prescribir espacios públicos cerrados 100\% libres de humo de tabaco.

La sociedad civil, en voz de varios actores, debatió de manera pública y frecuente en diversos medios impresos y de radio y televisión, centrando sobre todo la discusión en los siguientes aspectos:

a) No existe prohibición de fumar, sólo se está regulando dónde hacerlo. Desde hace más de cinco años, en los vuelos aéreos dentro de todo el territorio nacional está prohibido fumar, y sin embargo, la gente no ha dejado el tabaco, y nunca hubo amparo alguno que procediera contra esa medida.

b) Reglamentar que se prohíba fumar en espacios cerrados de restaurantes, bares, discotecas, busca fundamentalmente proteger a los trabajadores de dichos lugares, quienes deben laborar por espacio de ocho horas inhalando de manera constante el humo de la gente que fuma.

\section{g) Propiciar la coalición}

Lograr que las diferentes agrupaciones de la sociedad civil convergieran y encontraran puntos comunes a impulsar propició que de manera conjunta se pudieran establecer estrategias y se evaluaran los alcances y avances de las acciones emprendidas para impulsar las medidas legislativas.

Se realizaron reuniones frecuentes entre integrantes de ACTA, FIC y el Consejo Mexicano Contra el Tabaquismo para establecer la ruta crítica y la posición que se debía adoptar. Prueba de ello fue el hecho de que a pesar de que un grupo importante pensaba que la interferencia de la IT había logrado minar la Ley General para el Control del Tabaco, en respeto a la opinión de otro grupo importante que señalaba que era mejor contar con un marco legal específico que pudiera ser modificable en el mediano plazo, se tuvo el acuerdo de no interferir ni opinar de manera pública en contra de dicha ley, para que ésta fuera susceptible de aprobarse.

Ha sido contundente el papel que las diversas agrupaciones han tenido, pero sobre todo la unidad de ellas y la distribución de los quehaceres y responsabilidades, para evitar duplicidades y dispendio de recursos.

\section{Conclusiones y retos}

México cuenta hoy con avances importantes en materia del control del tabaco. Si bien aún queda mucho por avanzar, lo logrado en el último año abre esperanzas de que es factible impulsar el CMCT a plenitud.

\section{Ley de Protección a los No Fumadores} en el Distrito Federal

En el Distrito Federal, a un mes de la entrada en vigor (mayo de 2008), ha sido notable cómo la sociedad capitalina está cumpliendo con el marco normativo. Es de resaltar el enorme grado de cumplimiento de la Ley, sobre todo en lo que respecta a los espacios $100 \%$ libres de humo en restaurantes, bares, discotecas, boliches y billares, sin que hasta el momento se haya tenido que aplicar sanción alguna. Este hecho refuerza lo que las diversas encuestas de opinión han mostrado, que 7 de cada 10 personas fumadoras apoyaban la medida de establecer espacios 100\% libres de humo de tabaco.

Un hecho relevante es la respuesta inmediata que las autoridades locales de la secretaría de Salud y de la Consejería Jurídica del Gobierno de la Ciudad de México han tenido, publicando casi de manera inmediata tanto 
la Ley como su Reglamento respectivo, así como el hecho de que inmediatamente después de su aprobación, el Jefe del Gobierno manifestó públicamente su acuerdo con dicha normativa.

Si bien el Partido Alternativa Social Demócrata ha impulsado alrededor de 1800 amparos, sin que hasta el momento se conozca que alguno les haya sido otorgado, la gran mayoría de los más de 2 millones de fumadores que existen en el Distrito Federal han puesto su voluntad y empeño en cumplir con la normativa. Es indudable que se requiere consolidar la norma y propiciar su cumplimiento permanente y constante, para lo cual es indispensable mantener acciones de concientización y denuncia ciudadana.

\section{Ley General para el Control del Tabaco}

A pesar de haber sido aprobada la Ley el pasado 26 de febrero de 2007, hasta la escritura de este artículo aún no había sido publicada por el Ejecutivo, lo cual da un margen mayor para la interferencia por parte de la IT.

De igual forma, queda pendiente el hecho de la reglamentación respectiva y es notorio cómo en algunos temas se puede notar la intromisión de la IT, como en los tiempos señalados para la incorporación de pictogramas en las cajetillas y paquetes de los productos de tabaco, donde el plazo se puede alargar hasta 18 meses, a pesar de que nuestro país está ya a destiempo con el cumplimiento del CMCT.

Queda pendiente impulsar en el corto plazo modificaciones a dicha ley o a sus reglamentos respectivos, sobre todo en el sentido de lo siguiente:

a) Espacios 100\% libres de humo de tabaco: si bien la ley contempla que en los espacios cerrados puedan destinarse áreas aisladas específicas para fumadores, es importante que quede claro que en éstas no deberá existir ninguna prestación de servicio de alimentos o bebidas. Es necesario impulsar el que todas las entidades cuenten con leyes estatales donde se establezcan espacios públicos cerrados $100 \%$ libres de humo de tabaco.

b) Empaquetado y etiquetado: es la regulación que más apremia en virtud de los tiempos que se especifican en la ley. Requiere que el Ejecutivo, a través de la Secretaría de Salud, publique casi de manera inmediata la regulación respectiva. Es importante que nuestro país cuente con un banco de imágenes y de advertencias sanitarias, así como que pueda lograr establecer éstas para cada grupo objetivo consumidor.

c) Publicidad, promoción y patrocinio: la ley abre la posibilidad de realizar publicidad en áreas frecuentadas exclusivamente por mayores de edad y en puntos de venta, por lo que se hace fundamental llevar a cabo el análisis jurídico de constitucionalidad para proponer en el corto plazo restricciones mayores hasta alcanzar la prohibición completa. Queda claro que la IT ha dirigido de manera más que elocuente su publicidad hacia menores de edad, en clara contravención del marco regulatorio vigente y bajo la aprobación de la Cofepris.

d) Distribución, venta y suministro: es uno de los rubros en donde, a pesar de existir prohibiciones para la venta a menores y de manera unitaria, éstas son violadas de manera diaria en todo el país. Ello demuestra que mientras la responsabilidad de la vigilancia siga estando de manera centralizada en las autoridades sanitarias y no se den facultades para que las autoridades locales municipales y delegacionales puedan actuar en ese sentido, seguirá siendo una materia pendiente de cumplir.

e) Comercio ilícito. es importante establecer los criterios y políticas regionales a seguir, en donde se debe de considerar como temática relevante las ventas que de manera libre e indiscriminada se realizan a través de las tiendas libres de impuestos.

f) Vigilancia sanitaria: señalábamos ya la necesidad de establecer la coordinación necesaria para dotar de mayores atribuciones en cuanto a la vigilancia al ámbito estatal, municipal y delegacional.

g) Política fiscal: si bien recientemente existió un incremento sustancial en el impuesto especial al producto (IEPS), la realidad es que se deben impulsar y realizar los estudios económicos que conlleven a establecer mayor impuesto al producto. De igual manera se deberá discutir en el mediano plazo respecto de la posibilidad de que las entidades federativas establezcan impuestos especiales por la venta o consumo del cigarro.

Pese a lo anterior, debemos señalar claramente el que es indudable que el poderío económico y político que la IT tiene en el país es una amenaza constante para los avances que el control del tabaco requiere desde el punto de vista de la salud pública.

Por ello es fundamental no detenerse ante los recientes logros y continuar impulsando el CMCT y su aplicación inmediata en nuestro país, así como mantener un monitoreo constante de las acciones que la IT emprenda para lograr proteger a nuestras generaciones futuras de las múltiples causas de enfermedad y muerte que el tabaco ocasiona. 


\section{Referencias}

I. Barnoya J, Glantz S. Tobacco industry success in preventing regulation of secondhand smoke in Latin America: the "Latin Project". Tobacco Control 2002; I I:305-3|4.

2. Secretaría de Salud. Encuesta Nacional de Adicciones 1988. México, DF: SSA, 2002.

3. Instituto Nacional de Estadística, Geografía e Informática y Secretaría de Salud (SSA). Encuesta Nacional de Adicciones 2002. México: INEGI,

2004. Disponible en: www.consulta.com.mx/interiores/99_pdfs/I5_otros_ pdf/ENA.pdf.

4. Organización Mundial de la Salud. Encuesta Mundial de Tabaquismo y Juventud.

5. Organización Mundial de la Salud, Convenio Marco Para el Control del Tabaco, 2003. OMS, 2003.

6. Decreto por el que se aprueba el Convenio Marco de la OMS para el control del tabaco, adoptado en Ginebra Suiza, el 2I de mayo de 2003. Publicado en el Diario Ofcial de la Federación el 12 de mayo de 2004. México, 2004.

7. Reporte Financiero Anual de Grupo Carso al 31 de diciembre de 2006. http://www.gcarso.com.mx/GCarso/Esp/Relación+con+Inversionistas/ Información+Financiera/Reportes+Anuales.htm

8. Kuri-Morales PA, González-Roldán JF, Hoy MJ, Cortés-Ramírez M. Epidemiología del tabaquismo en México. Salud Publica Mex 2006;48(supl I):S9I-S98.

9. Organización Mundial de la Salud. Conferencia de los estados Parte en el Convenio Marco de la OMS para el control del tabaco, segunda reunión. Directrices sobre la protección contra la exposición del humo de tabaco. Bangkok, Tailandia, julio 2007. 